\title{
Processing of global, but not local, motion direction is deficient in schizophrenia
}

\author{
Y. Chen ${ }^{\text {a,b,* }}$, K. Nakayama ${ }^{\text {b }}$, D. Levy ${ }^{\text {a }}$, S. Matthysse ${ }^{\text {a }}$, P. Holzman ${ }^{\text {a,b }}$ \\ ${ }^{a}$ Department of Psychiatry, Harvard Medical School/McLean Hospital, USA \\ ${ }^{\mathrm{b}}$ Department of Psychology, Harvard University, USA
}

Received 14 December 2001; received in revised form 6 March 2002; accepted 12 March 2002

\begin{abstract}
Visual motion processing is compromised in a substantial proportion of schizophrenic patients, but precise neural mechanisms underlying the motion-processing deficit have not yet been elaborated. The visual motion pathway includes a local and a global processing stage, each of which has distinct neural substrates. Here, we attempt to identify the stage(s) that are implicated in impaired motion processing of schizophrenia - local, global, or both. For schizophrenia patients $(n=23)$ and normal controls $(n=26)$, we measured (1) the thresholds for detecting the motion direction of a random dot pattern, a task that requires global motion processing, and (2) the thresholds for detecting the motion direction of a grating, a task that requires only local motion processing, using psychophysical methods. Schizophrenia patients showed elevated thresholds for detecting the direction of coherent motion, particularly for the high dot-density target. In contrast, schizophrenia patients showed normal thresholds for detecting the direction of motion of a grating. The results indicate that the global, but not the local, processing stage of the visual motion system is compromised in schizophrenia patients, thus implicating motion-sensitive brain areas that possess large receptive fields for spatial and temporal integration, such as Middle Temporal Area/Medial Superior Temporal Area.
\end{abstract}

(C) 2002 Elsevier Science B.V. All rights reserved.

Keywords: Vision; Coherent motion; Smooth pursuit; Mental diseases; Psychophysics; Schizophrenia

\section{Introduction}

In schizophrenia, many cognitive, affective, motor and perceptual behaviors are impaired. Some of these impairments directly point to the brain processes that underlie the mental disorder. One example is the impairment of smooth pursuit eye movements (SPEM), which appears in many schizophrenic patients and in a

\footnotetext{
* Corresponding author. Mailman Research Center, Room 123D, McLean Hospital, 115 Mill Street, Belmont, MA 02478, USA. Tel.: +1-617-855-3615; fax: +1-617-855-2778.

E-mail address: ychen@wjh.harvard.edu (Y. Chen).
}

proportion of their first-degree relatives (Diefendorf and Dodge, 1908; Holzman et al., 1973, 1974; Clementz and Sweeney, 1990; Iacono et al., 1992; Levy et al., 1993; Friedman et al., 1995). Smooth pursuit is a complex behavior that involves many sensory, cognitive, and oculomotor processes. The requirement to detect a moving target of interest generally initiates smooth pursuit eye movement. Assessing the functional integrity of the motion processing system therefore assumes importance for probing into the brain mechanisms underlying the smooth pursuit impairment in schizophrenia. Visual motion detection and movement-related detection are compromised in schizophre- 
nia patients (Wertheim et al., 1985; O'Donnell et al., 1996; Stuve et al., 1997; Chen et al., 1999a,b). Here, we attempt to identify the stage of motion processing, global or local, that is deficient in schizophrenia.

It is generally believed that motion processing begins with neural processing in retina. The neural pathway of motion processing continues to the lateral geniculate nucleus and to the striate cortex (V1). Up to this point, neural processing of visual information is not motion-specific, that is, other aspects of visual signals, such as form, contrast, and color, are processed in the same brain areas. The motion signals are then projected from V1 to the extrastriate cortex, where two of its more than 20 areas, middle temporal area (MT) and medial superior temporal area (MST), process motion-specific information. In some parts of MT, motion processing is similar to that in V1 and focuses on the local signals, and in other parts, however, motion processing becomes global (e.g., Born and Tootell, 1992). In the local stage, motion detection occurs when a moving target activates many independent motion-sensitive units that correspond to different spatial locations. The receptive field of these units is usually small and thus responds to only a restricted part of the visual stimulus (e.g., Sclar et al., 1990). The responses of these neural units at the local stage could be used to represent target movement if the motion signals were uniform across space. For example, the direction of a moving grating can be ascertained by viewing only a portion of it, since all parts of the grating move in the same direction and at the same speed and as a whole. The neural units at the local stage, however, are unable to generate a coherent motion percept if motion signals are not identical at different spatial locations. In this instance, global processing is required. The global motion processing comprises neural activity in other parts of MT and in MST, where neural units have large receptive field sizes (e.g., Sclar et al., 1990). Thus, these neural units are capable of integrating dispersed motion signals across space and of generating the perception of a coherent whole by taking into account the fact that the motion signals are usually spatially varied (e.g., Tanaka et al., 1986; Born and Tootell, 1992). For example, in a random dot pattern, the dots move independently of each other, and the neural activities that respond to the individual moving dots at the local stage therefore differ from each other. To generate a motion percept of the coherent whole in a random dot pattern, motion processing at the global stage must combine the responses from the local-stage neural units that are activated by individual moving dots. The global direction of the random dot pattern can be judged only by apprehending at least a significant portion of the whole target (Nakayama and Silverman, 1984; Williams and Sekuler, 1984). These local and global stages of motion processing show different functional properties (Adelson and Movshon, 1982; Williams and Sekuler, 1984; Nakayama, 1985). This distinction between the local and the global stages is mainly, but not exclusively, in the spatial domain.

With respect to schizophrenia, an important question is to identify the specific stage, local or global, of motion processing that is responsible for the motionprocessing deficit. Answering this question may provide information not only about the functional characteristics of the affected motion processing system, but also about the neural substrates underlying the motion detection deficit, and hence about the smooth pursuit impairments and the pathophysiology of schizophrenia.

In this study, we attempt to identify the motion processing stage(s) that are implicated in schizophrenia, using paradigms widely employed in physiological and psychophysical studies. Specifically, we determined the response effectiveness with which schizophrenic patients detect global motion direction signals (coherent motion) and local motion direction signals (movement of gratings).

\section{Methods}

\subsection{Subjects}

Twenty-three schizophrenic patients participated in this study. These patients were discharged during the past year from a psychiatric hospital and met DSM-IV criteria for schizophrenia or schizoaffective disorder. Consensus diagnoses were made independently, and blind to the experimental design and results, by experienced clinicians based on a review of a standardized interview (Structured Clinical Interview for the DSM-IV, Spitzer et al., 1994), conducted by trained interviewers, and an evaluation of all available hospital records. All patients were receiving antipsy- 
chotic medication [mean daily chlorpromazine dose equivalent: $567 \mathrm{mg}(\sigma=386 \mathrm{mg})$ ]. The average Brief Psychiatric Rating Scale (BPRS) score (Overall and Gorham, 1962) of the patients was $39.2(\sigma=12.1)$, and the average duration of illness was 14.9 years ( $\sigma=6.8$ years), indicating that these were chronically ill patients, in various stages of partial remission.

Twenty-six nonpsychotic controls participated this study. They were recruited from a large medical outpatient clinic. None of the normal controls met DSM-IV criteria for a psychotic condition (lifetime) or for schizotypal or schizoid personality disorder, based on a standardized interview (Kendler, 1989). Seventeen of these control subjects had no diagnosable clinic condition. Three had migraine headaches and two suffered from asthma. Only one control subject was receiving antidepressant and one antianxiety agent (Xanax). All others were either unmedicated, or taking asthma medication, or nonsteroidal anti-inflammatory medications (such as naprosyn and aspirin), or estrogen supplements.

Visual acuity of the patients and normal controls was not assessed directly because motion processing uses the spatial frequency information that is not clearly related to this measurement. Instead, we used contrast detection of moving gratings to evaluate the subjects' visual sensitivity to motion signals. The patient and the normal control groups were matched for age, sex, education, and socioeconomic status (Table 1). Written informed consent in accord with the IRB guidelines of McLean Hospital and Harvard University was obtained from all participants prior to testing.

Table 1

Demographic characteristics of the sample

\begin{tabular}{|c|c|c|c|c|}
\hline Group & $\operatorname{Age}^{\mathrm{a}}$ (years) & $\operatorname{Sex}^{\mathrm{b}}$ & $\mathrm{SES}^{\mathrm{c}}$ & $\begin{array}{l}\text { Education } \\
\text { (years) }\end{array}$ \\
\hline \multirow{2}{*}{$\begin{array}{l}\text { Schizophrenic } \\
\quad(n=23)\end{array}$} & 39 (7) & $14 \mathrm{~F}, 9 \mathrm{M}$ & $\mathrm{I}-22 \%$ & $15(2)$ \\
\hline & & & $\begin{array}{l}\text { II }-48 \% \\
\text { III-30\% }\end{array}$ & \\
\hline \multirow{2}{*}{$\begin{array}{l}\text { Normal control } \\
\qquad(n=26)\end{array}$} & 39 (13) & $17 \mathrm{~F}, 9 \mathrm{M}$ & I-19\% & $14(2)$ \\
\hline & & & $\begin{array}{l}\text { II }-46 \% \\
\text { III- } 35 \%\end{array}$ & \\
\hline
\end{tabular}

${ }^{\mathrm{a}}$ Mean (standard deviation).

${ }^{\mathrm{b}} \mathrm{F}=$ female, $\mathrm{M}=$ male.

c SES-socio-economic status, based on Hollingshead and Redlich two-factor index (Hollingshead, 1965).

\subsection{Procedure}

There were three motion tasks: (1) detection of the direction of coherent (global) motion stimulus, (2) detection of the direction of a local motion stimulus, and (3) detection of the presence of a motion target.

\subsection{Coherent motion}

The target for detection of coherent motion was a random dot pattern, displayed on a computer screen. The signal component was an array of dots moving coherently in one direction (left or right) and the noise component was another array of dots moving in random directions (Newsome and Pare, 1988). These two components were interleaved spatially and temporally within a rectangular window $(8 \times 20 \mathrm{deg})$. The dots were small $(2 \times 2 \mathrm{~min}$ arc $)$ and white, and were presented on an otherwise black background (Fig. 1). Target movement was generated by positional displacement of the individual dots. The displacement was equivalent to $10 \%$ and occurred in every subsequent display frame.

Subjects were required to judge the direction (left or right) of motion of the signal component. The percentage of signal dots in the pattern, called motion coherence, represents the task-difficulty level. The smaller the proportion of signal dots (i.e., the lower the coherence), the more difficult it is to perceive the direction of motion. The critical measure is the minimum percentage of signal dots (i.e., the minimum coherence level) at which the performance of a subject reaches the criterion of $75 \%$ correct in judging the movement direction of the random dot pattern. This percentage level is defined as the threshold. We measured the thresholds for all subjects for each of three dot densities: 50, 100, and 200 dots (i.e., $0.31,0.62$, and $1.24 \mathrm{dots} / \mathrm{deg}^{2}$, respectively). Fig. 2 shows an example of the psychometric function generated by this paradigm. The psychometric function takes into account each subject's percent correct scores at all coherence levels, not just those at one coherence level, in determining a threshold. It is these thresholds at each of the three dot densities (50, 100 , and 200 dots) for each subject that provide the dependent variables analyzed in the Results.

The stimuli for all tasks were generated on a Macintosh computer (Quadra 610). Subjects viewed the computer screen and, when ready, started a 


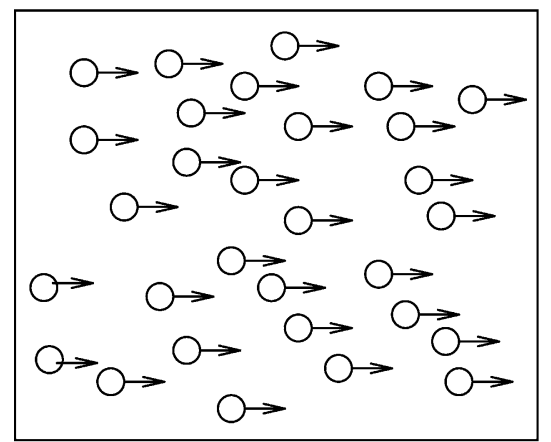

signal: $100 \%$

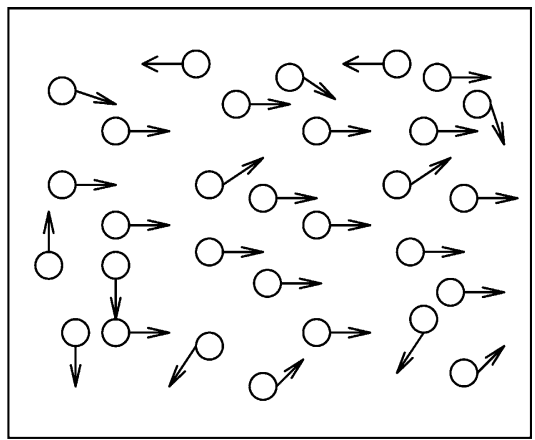

signal: $50 \%$

Fig. 1. Stimulus configuration for detecting the direction of coherent motion. The two components of stimulus are (1) coherent motion dots or signal dots (moving in one direction, left or right), and (2) random motion dots or noise dots (moving in random directions). The percentage of the coherent motion dots is defined as the motion coherence level and is an index of signal strength. A $100 \%$ coherence level means that all dots in the stimulus move in the same direction; a 50\% coherence level means that half of the dots move in the same direction and the other half move in random directions. The higher the coherence level, the easier the task of judging the direction of the dots, and conversely, the lower the coherence level, the more difficult the task of judging direction of the dot flow.

session by pressing a key. In the coherent motion task, each session contained 80 trials, which were evenly divided but randomly distributed across the

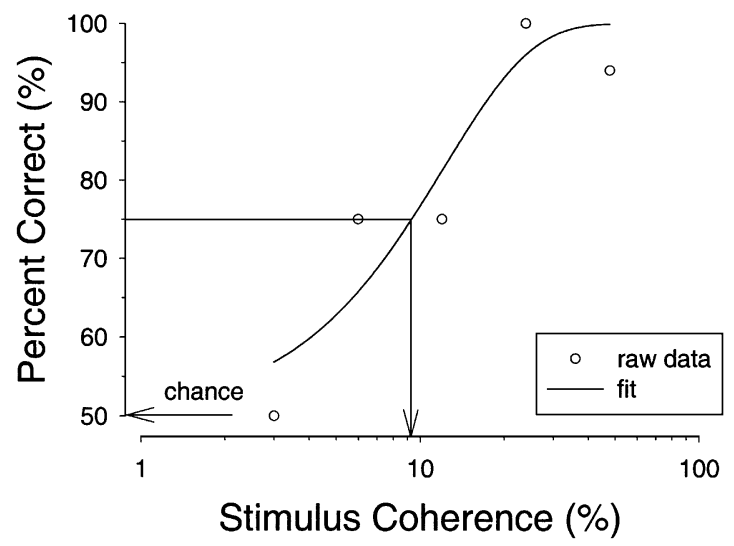

Fig. 2. Psychometric function for determining thresholds for detecting coherent motion. The five coherence levels of the random dot presentations $(3 \%, 6 \%, 12 \%, 24 \%$, and $48 \%)$ are represented on a logarithmic scale along the abscissa; the accuracy with which a subject determines the direction of movement of the signal dots is represented on a linear scale along the ordinate. The five data points represent the percent correct judgments at the five coherence levels presented. The most difficult coherence level is at $3 \%$, and the percent correct is at a chance level; the easiest levels are at $24 \%$ and $48 \%$ where percent accuracy scores are over $90 \%$. The data are fit by a form of Weibull function: $y=100-50 \exp \left[-(x / \alpha)^{\beta}\right]$. A threshold is determined from the fitted curve. The threshold in this illustration, set at the $75 \%$ level of accuracy, is at a coherence level of $9.2 \%$. five motion coherence levels. The percentage of signal dots in the random dot pattern varied across trials according to the method of constant stimuli (among 3\%, 6\%, 12\%, 24\%, and 48\% coherence). During each trial the random dot pattern was presented at the center of the visual field. The movement of the signal dots was either to the left or to the right, varying randomly from trial to trial, and lasting 750 ms. After viewing the dot flow stimulus in each trial, subjects indicated their judgment about the direction of motion (left or right) by pressing one of two designated keys. No feedback for the correctness of the response was provided except in practice sessions. Inter-trial intervals were varied randomly from 500 to $1000 \mathrm{~ms}$. To prevent focusing on any single dot rather than on the whole pattern, and to minimize a shift in fixation, dot lifetime was limited to $90 \mathrm{~ms}$ (6 frames). The relatively long duration was used to accommodate a request from some subjects who were unable to perform the task with a shorter duration. A small fixation circle was presented continuously in the center of the field.

Under the condition of $100 \%$ coherence, detection of coherent motion can be a local motion task because every dot in the random dot pattern moves in the same direction. For that condition, motion signals from a single dot are the same as those from the whole pattern of random dots. Local processing of motion signals is thus sufficient for representing the move- 
ment of the random dot pattern. In this sense, the detection of coherent motion at a high coherence level can serve as another local motion comparison.

\subsection{Detection of the direction of a local motion} stimulus

The target for assessing the direction of a local motion stimulus was a vertical grating (Fig. 3). The spatial luminance distribution of the grating was a sinusoidal waveform (spatial frequency: 0.5 cycles/ deg). The temporal modulation was set at $5 \mathrm{~Hz}$, which yielded target movement of $10 \% \mathrm{~s}$, either to the right or to the left. The target was presented within a circular window with a diameter of $10^{\circ}$ of visual angle.

The task for detecting the direction of a local motion stimulus was to judge the direction of motion of gratings (left or right). The dependent measure was

\section{Direction discrimination}

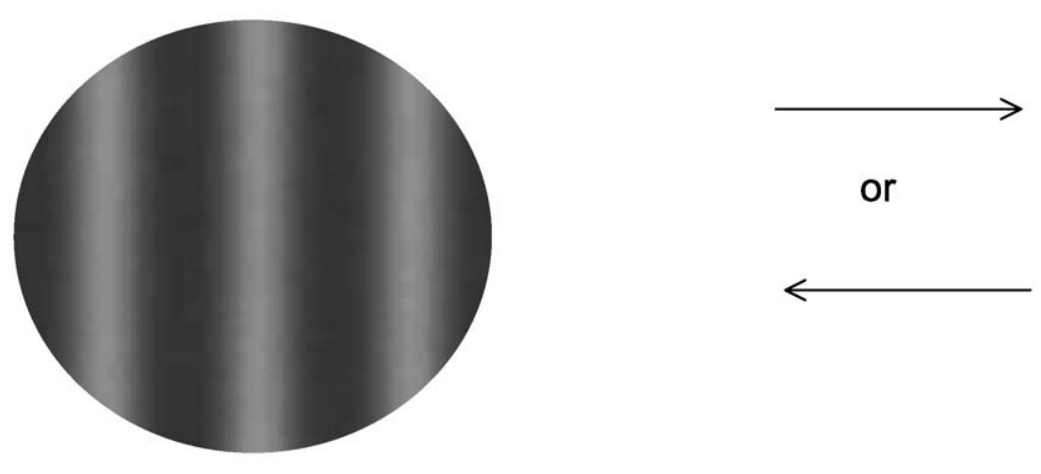

Which direction do gratings move in

\section{Contrast detection}
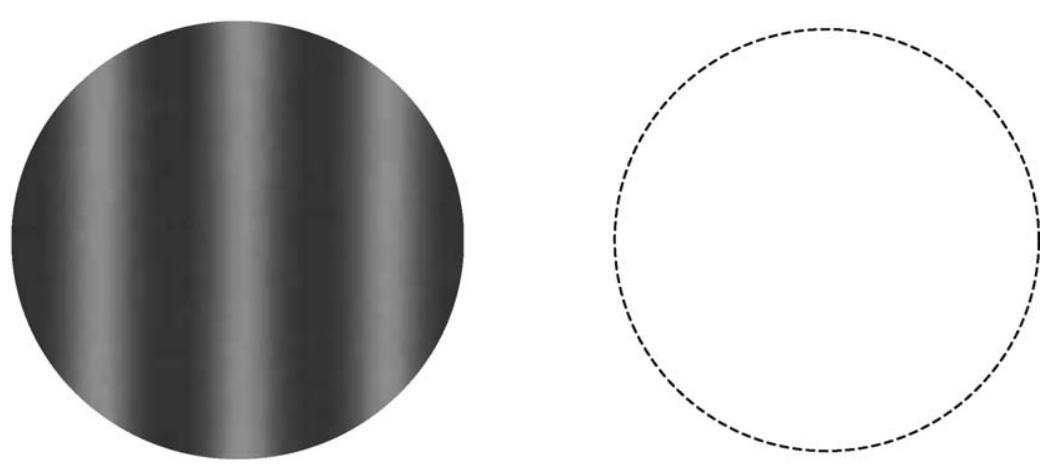

\section{Which interval contains gratings}

Fig. 3. Stimulus configurations for detecting the movement direction of a local motion stimulus (top) and for detecting the presence of a motion target (bottom). 
the minimum amount of contrast necessary to identify the correct direction of movement at $79 \%$ accuracy level. The contrast of the gratings was set initially at $1.5 \%$, a level adequate to detect the direction of motion. The level of contrast varied from trial to trial according to a two-alternative forced-choice staircase method (1-up-3-down). Specifically, the contrast decreased by $5 \%$ of the current level if three correct responses were made in a row, and increased by $5 \%$ of the current level if one incorrect response was made. Twelve reversals of staircase direction terminated an experimental session. The contrast levels at all reversals, except for the first one, were averaged to produce a threshold. Compared with the method of constant stimuli used in detecting the direction of coherent motion, the staircase method requires less testing time.

Subjects made judgments about the direction of motion for each target presentation by pressing one of two designated keys (left or right). The target movement was either to the left or to the right, and varied randomly from trial to trial. Inter-trial intervals were $1000 \mathrm{~ms}$. Each grating was presented on the screen for $300 \mathrm{~ms}$. A fixation cross was provided at the center of the field.

\subsection{Detection of the presence of a moving target}

The subjects' task was to indicate at which of the two temporal intervals (first or second) in a trial the target was present. The target for detecting the presence of a moving target was the same grating used in the direction discrimination task (Fig. 3). The detection task provides an index of the amount of contrast that is required to detect the presence of a moving target, independently of judging any motion attribute of the target, such as its direction of movement or velocity.

During each trial, two temporal intervals were presented, one containing the target and the other a blank area whose luminance level was the same as that of the target. The target could appear at either the first or the second interval, randomized across the trials. The dependent measure was the minimum contrast that allowed detection of the moving target at a $79 \%$ accuracy level. The contrast of the gratings was set initially at $1.5 \%$, a level adequate to detect the presence of the moving gratings. The contrast levels were then varied from trial to trial according to a twoalternative forced-choice staircase method (1-up-3- down). Specifically, the contrast was decreased by $5 \%$ of current level if three correct responses were made in a row, and was increased by $5 \%$ of current level if one incorrect response was made. Twelve reversals of the staircase direction terminated an experimental session. The contrast levels of all reversals, except for the first one, were averaged to produce a threshold.

By these customized procedures, we managed to administer all three tasks within a single one-hour session. Detailed instructions and adequate practice for each task were administered prior to formal data collection. Short breaks were provided during the session when necessary.

\section{Results}

\subsection{Coherent motion}

As described above, we determined thresholds for detecting coherent motion at each of the three dot densities, based on a psychometric function for each subject. As a threshold, we used the motion coherence level that yielded $75 \%$ accuracy in performance. Percent correct scores for judging the direction of motion were fitted as a function of the motion coherence level (see Fig. 2). This graph, commonly termed a psychometric function, was plotted for each of the three dot densities, based on the subjects' responses in all trials. Fig. 4 presents a scatter plot of the thresholds of each schizophrenia subject and each nonpsychiatric control. At all three dot-densities tested, the schizophrenia subjects performed more poorly (i.e. had a higher threshold value) than the controls $\left(t_{(47,1)}=2.52, p<0.05\right.$ for 50 dots; $t_{(47,1)}=$ $2.29, p<0.05$ for 100 dots; $t_{(47,1)}=2.67, p<0.05$ for 200 dots). While occurring under all dot densities, the magnitude of the mean difference in threshold between groups was greatest when the 200-dot target was used. Analysis of variance with two subject groups and 3-dot densities confirmed that the schizophrenia group had significantly elevated thresholds, compared with the normal control group $\left(F_{1,146}=\right.$ $16.43, p<0.001)$. Although the group $\times$ dot density interaction was not statistically significant, a ratio comparison indicates that the largest group difference occurred in the 200-dot condition (see Table 2). This largest group difference was accompanied by a large 


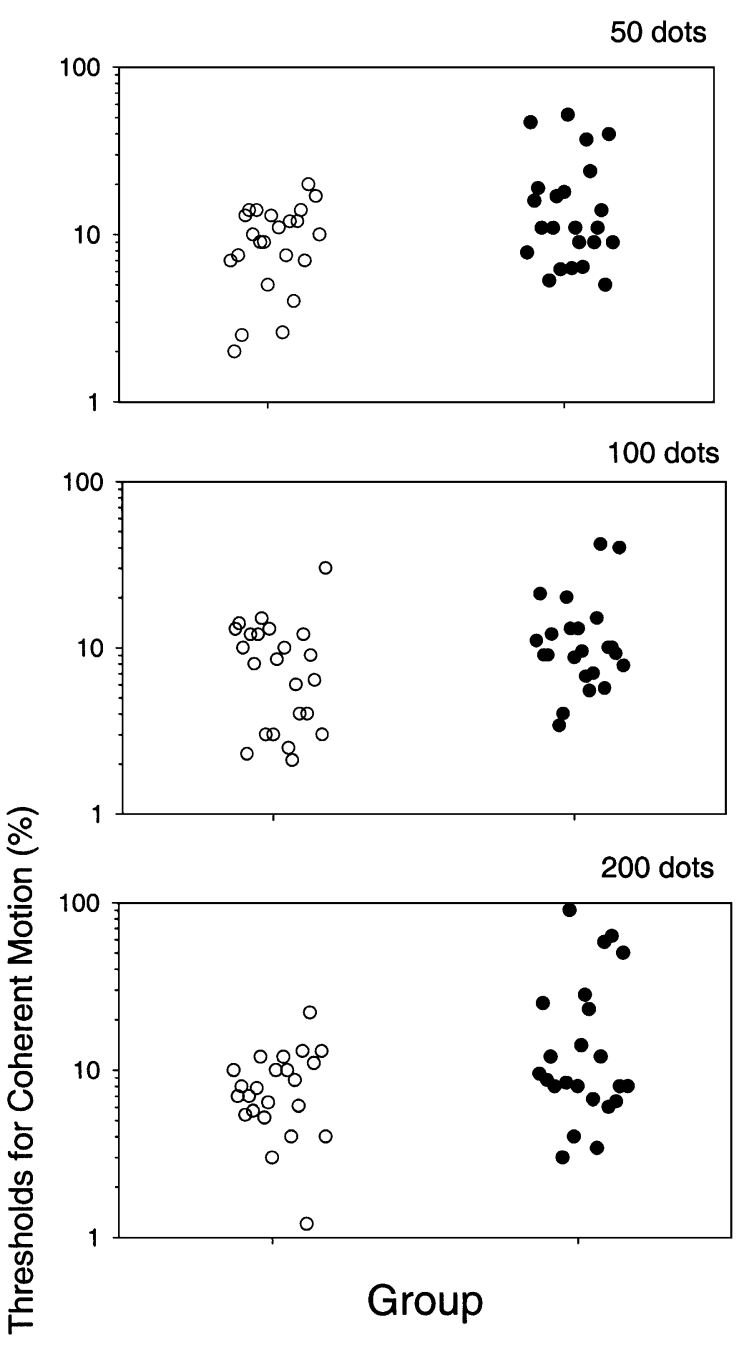

Fig. 4. Scatter plot of coherent (global) motion thresholds of individual subjects in the two groups. The upper, middle, and lower panels correspond to the results obtained under 50-, 100-, and 200dot density conditions, respectively. In each panel, the data points represent the thresholds ( $y$ axis, logarithmic scale) of individual subjects, that is, the coherence level at which a subject obtained $75 \%$ accuracy in judging direction of the dot movement flow. The filled circles represent schizophrenic patients and the open circles represent normal controls.

variance in the patient group, which effectively reduced the effect size between the different performances of the two groups. The thresholds and standard deviations for both groups are provided in Table 2.

For detailed comparisons, the percent correct scores for both groups are provided in Table 3. It is
Table 2

Means and standard deviations of coherent motion thresholds (percent of coherence)

\begin{tabular}{|c|c|c|c|c|c|c|}
\hline \multirow[t]{2}{*}{ Group } & \multicolumn{2}{|c|}{50 dots } & \multicolumn{2}{|c|}{100 dots } & \multicolumn{2}{|c|}{200 dots } \\
\hline & $\overline{\mathrm{SZ}}{ }^{\mathrm{a}}$ & $\overline{N^{b}}$ & $\overline{\mathrm{SZ}}$ & $\mathrm{NC}$ & $\overline{\mathrm{SZ}}$ & $\mathrm{NC}$ \\
\hline Threshold & $\begin{array}{l}17.0 \\
(13.3)\end{array}$ & $\begin{array}{l}9.6 \\
(4.6)\end{array}$ & $\begin{array}{l}12.7 \\
(8.3)\end{array}$ & $\begin{array}{l}8.4 \\
(4.3)\end{array}$ & $\begin{array}{l}20.1 \\
(23.0)\end{array}$ & $\begin{array}{l}8.0 \\
(4.3)\end{array}$ \\
\hline Ratio (SZ/NC) & 1.75 & & 1.44 & & 2.39 & \\
\hline Effect size & 1.0 & & 0.7 & & 1.0 & \\
\hline
\end{tabular}

${ }^{a}$ Schizophrenia.

b Normal control.

noteworthy that the patient group has significantly lower percent correct scores principally when judging the $6 \%$ coherent motion target $(p<0.05$ for all three dot conditions; see Table 3 for a complete comparison). Under the 200-dot condition, for example, the means of the percent correct score are $64 \%$ and $72 \%$ for the patient and the normal control groups, respectively, the largest group difference observed among all the dot-density conditions and the motion coherence levels tested. At 3\% coherence, the most difficult condition, both groups were unable to detect reliably the direction of coherent motion: the performance of both groups hovering just above chance levels (53$60 \%$ correct). At $24 \%$ and $48 \%$ motion coherence, the two easiest conditions, both groups were equally able to detect the direction of coherent motion; the performance of both groups equalled or exceeded the $90 \%$ accuracy level.

\subsection{Detection of the direction of a local motion stimulus}

The thresholds for detecting the direction of motion of a grating were $0.0018(\sigma=0.0009)$ for the

Table 3

Means and standard deviations of percent-correct scores in coherent motion task

\begin{tabular}{|c|c|c|c|c|c|c|}
\hline \multirow[t]{2}{*}{ Coherence $(\%)$} & \multicolumn{2}{|l|}{50 dots } & \multicolumn{2}{|c|}{100 dots } & \multicolumn{2}{|c|}{200 dots } \\
\hline & $\mathrm{SZ}^{\mathrm{a}}$ & $\mathrm{NC}^{\mathrm{b}}$ & $\overline{\mathrm{SZ}}$ & $\mathrm{NC}$ & $\overline{\mathrm{SZ}}$ & $\mathrm{NC}$ \\
\hline 3 & $53(13)$ & $57(18)$ & $58(14)$ & $63(13)$ & $60(12)$ & $57(17)$ \\
\hline 6 & 58 (19) & $65(17)$ & $66(16)$ & $70(13)$ & $64(14)$ & $72(12)$ \\
\hline 12 & 77 (15) & 79 (13) & 77 (13) & 79 (13) & $78(20)$ & $80(14)$ \\
\hline 24 & 89 (10) & $95(6)$ & $92(12)$ & $94(7)$ & $90(13)$ & $90(12)$ \\
\hline 48 & $96(8)$ & $96(7)$ & $98(5)$ & $98(5)$ & $95(9)$ & $97(5)$ \\
\hline
\end{tabular}


normal controls and for the schizophrenia patients $0.0020(\sigma=0.0007)$. The two groups did not differ significantly in this measure $\left(t_{(47,1)}=1.17, p>0.20\right)$. Fig. 5 presents the contrast thresholds for the two groups.

\subsection{Detection of the presence of a motion target (contrast detection)}

The thresholds for detecting the presence of moving targets (contrast detection) were $0.0035(\sigma=0.0021)$ for the normal controls and for the schizophrenia patients $0.0025(\sigma=0.0014)$. The two groups did not differ between the schizophrenia patient and the normal control groups $\left(t_{(41,1)}=1.67, p>0.10\right)$. Fig. 6 presents the contrast thresholds of the two groups.

There was no significant correlation between duration of illness and task performance in the patients. The patients' performance was also not correlated with the amount of antipsychotic medication prescribed (as measured by mean daily chlorpromazine dose equivalent). The Pearson product-moment correlation between BPRS score (a measure of illness severity) and the coherent motion threshold for the 200-dot density condition was statistically significant $\left(r_{200}\right.$, $\mathrm{BPRS}=0.55, p<0.05$ ). Between GAS score (another measure of illness severity) and the coherent motion

\section{Local Motion Task}

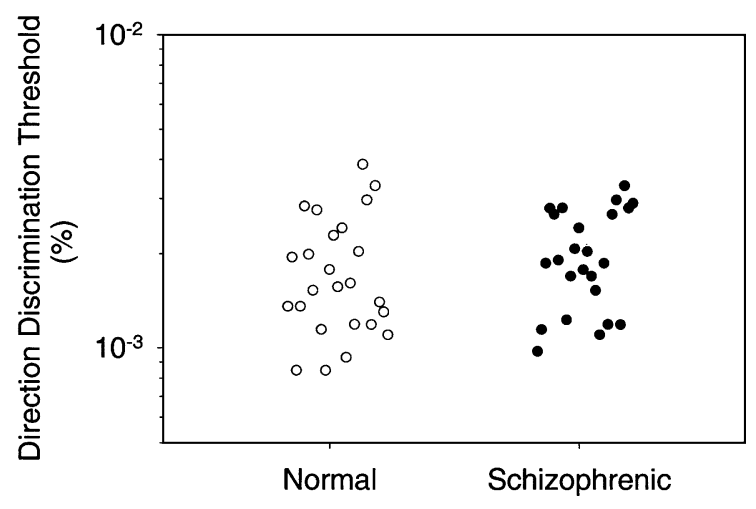

Fig. 5. Scatter plot of thresholds of individual subjects for detecting the direction of a local motion stimulus. Each point in the plot represents an individual subject's threshold of the contrast level required to detect the direction of moving gratings. Filled circles represent the schizophrenic patients and open circles the normal controls.
Contrast Detection

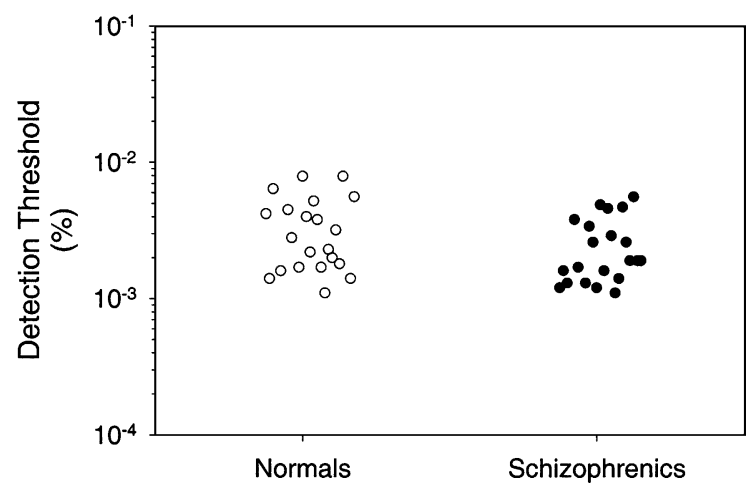

Fig. 6. Scatter plot of percent accuracy for the two subject groups for detecting the presence of a moving target. Filled circles represent the schizophrenic patients and open circles the normal controls.

threshold, the correlation was statistically significant at the 50- and the 200-dot density conditions $\left(r_{50}\right.$, $\left.\mathrm{GAS}=0.40, p<0.05 ; r_{200, \mathrm{GAS}}=0.39, p<0.05\right)$.

\section{Discussion}

Schizophrenia patients differed from nonpsychiatric controls on one of the two types of motion perception tasks employed in this study. The patients showed a selective deficit in detecting coherent motion of random dots, which requires global processing of the target. In this global motion detection task, motion information from many spatial and temporal locations must be combined in order to render a correct perceptual judgment about the direction of movement of the random dot pattern. In contrast, performance on tasks that require only local motion processing and only the detection of the presence of a moving stimulus did not distinguish the schizophrenia patients from the controls.

It would be ideal to use exactly the same stimuli and methods for the local and the global motion tasks. However, in order to tap into the separate processes for local and global motion, the task-design must take into account the different functional properties associated with the two tasks. Let us consider several factors that might have contributed to the differences in performance of schizophrenia patients in the global and local motion tasks. First, the random dot patterns 
contain a spectrum of spatial frequency components (i.e., both low and high) while the gratings contain only one low spatial frequency component $(0.5 \mathrm{cycle} /$ deg). The poor performance of schizophrenia patients cannot, however, be attributed to the presence of high spatial frequency components in the random dot pattern because at high coherence levels $(24 \%$ and $48 \%$ ); where the spatial frequency components are similar, both groups' performance was equivalent. Second, it is possible that the differences in performance reflect differences in target condition between the two tasks, inasmuch as the random dot motion task is performed at supra-threshold level and the grating motion one is performed at near-threshold level. This interpretation, however, is inconsistent with the results of a previous study (Chen et al., 1999b) in which velocity discrimination was examined using contrast as the principle dependent variable (i.e. at a near-threshold level); in that study, the performance was significantly deteriorated in schizophrenia patients. Third, it is possible that difference in psychophysical methods influenced the results insomuch as the thresholds for the global task were determined by the method of constant stimuli and the thresholds for the local motion task were determined by the staircase method. The normal performance of the patients in the local motion task, however, cannot be due to the use of the staircase method because, in previous studies (Chen et al., 1999a,b), schizophrenic patients showed deficient velocity discrimination when measured with the staircase method. Moreover, the performance in this study was compared between the two groups, rather than between the two tasks, making the methodological difference between the two tasks less important. A relevant point is that we used five coherence levels of a random dot pattern, ranging from low to high coherence, and global integration is required mainly at low coherence levels. The high coherence random dots can be considered to be a control condition for local motion processing. The random dot patterns with high and low coherences were equivalent in every respect except for the percentage of coherently moving dots. The normal performance of the patients at high coherence levels ( $\geq 90 \%$ correct) indicated that their difficulty at moderately low coherence level (i.e. 6\%) is due to the weak motion signals that need to be integrated by global motion processing.
The patients' normal performance in the local motion task, on the other hand, is not due to the task being too easy. For both groups of subjects, the task difficulty in detecting local motion was increased gradually until it reached the point that each subject was unable to perform the task (staircase method). This procedure has been effective in detecting performance differences between normal controls and schizophrenia patients in several other visual tasks, such as contrast detection (Slaghuis, 1998) and velocity discrimination (e.g., Chen et al., 1999a,b).

We therefore conclude that the specific deficiency on the coherent motion task at low coherence levels suggests that the global processing stage of the visual motion system is impaired in schizophrenia. The fact that both the local and global motion perception tasks require similar perceptual judgments - that is, to judge the direction of a target movement-indicates that the results do not represent a generalized cognitive deficit, such as would be involved in understanding the task requirements or in making decisions about judging direction.

As the density of the random dot pattern increases, both the local and global stages of motion processing are implicated to a greater extent. First, when more moving dots are present, the number of localized units that are activated increases, activating the local stage. Second, the demand for integrating the responses from the localized units is increased, activating the global stage. For the schizophrenic patients, the second explanation appears to be the more plausible one because, according to our data, the local stage is not abnormal in this population. Global motion processing becomes more important for high-density random dot patterns where a larger amount of information must be combined. In this study, the severest performance degradation and the largest performance variance in the schizophrenic patients occurred at the highestdensity condition (200 dots), suggesting that the global, but not local, processing of a large amount of motion information is compromised. The second severest performance degradation of the patients occurred at the 50-dot, rather than 100-dot, condition, suggesting that the dot-density effect is not simply monotonic, and further study is needed to delineate the complexity. Note that dot density negligibly affects motion detection using random dots, both in 
normal people (Downing and Movshon, 1988) and in animals with a damaged motion system (Rudolph et al., 1994). One interpretation for the dot density effect in schizophrenia is that the noise signal interferes with the integration of motion signals to a greater extent in the patients than in the normal controls; at the same coherence levels, the absolute number of noise dots is greater under high dot densities than under low dot densities. A separate study is needed to differentiate the noise from pure integration. In this connection, it is noteworthy that Rudolph and Pasternak (1999) showed that motion deficits in MT-damaged monkeys became permanent only when the moving stimuli were presented with noise.

Neurophysiological studies indicate that area MT, located at the junction of the occipital, temporal, and parietal lobes, is a primary site for motion processing (Dubner and Zeki, 1971). In the presence of motion signals, including those generated by a dynamic random dot pattern, neural units in MT are vigorously activated and encode motion attributes, such as direction and velocity (Rodman and Albright, 1989). Moreover, receptive field sizes are quite large for most neural units in this cortical area, which differentiate them categorically from many of their precursors, such as those in V1 (Sclar et al., 1990; Movshon and Newsome, 1996), and provide a necessary capacity for spatial integration of motion information. In human, several studies (e.g. Tootell et al., 1995; Beauchamp et al., 1997) showed that MT selectively responds to motion, rather than other types of, signals. A recent study compared the performance on the same motion perception tasks in a motion-blind patient and in a monkey with MT removal, and concluded that the motion processing deficit shown in the patient was attributable to damage to the human equivalent of area MT and its adjacent areas (Marcar et al., 1997). Furthermore, Vaina et al. (2001) reported that perception of the direction of global motion was selectively impaired in the visual field contralateral to the lesion in stroke patients whose lesions were in the occipitoparietal and parieto-temporal areas, which involved the human analogue of areas MT/V5 and MST. Patients with lesions in the occipito-temporal or anterior frontal areas, however, did not show these global motion direction impairments. In this respect, the impaired performance of schizophrenia patients in the global coherent motion task suggests that the motion-sensi- tive brain areas, such as MT, are compromised in schizophrenia.

While area MT is centrally implicated in motion processing, other brain areas are involved as well. Neural units in cortical areas (e.g., MST) as well as subcortical (e.g., superior colliculus and thalamus) areas respond to various motion stimuli, including random dot patterns (Celebrini and Newsome, 1994; Bender and Davidson, 1986; Collin and Cowey, 1980; Merabet et al., 1998). The behavioral responses from brain-damaged patients and monkeys also suggest that lesions in many brain areas, such as area MT, the adjacent area MST, and the fundus superior temporal (FST) visual area (Orban et al., 1995), lateral occipital gyri (Plant et al., 1993), superior colliculus (Collin and Cowey, 1980; Bender and Davidson, 1986) and even the cerebellum (Nawrot and Razzio, 1995), lead to deficits in motion processing. Recent functional brain imaging studies showed that motion processing indeed involves many motion-sensitive brain systems (Sunaert et al., 2000). The specificity of the processing stages for local and global motion signals continues upstream, along the motion pathway, at least to MST and FST (Berezovskii and Born, 2000). In schizophrenia, gross structural damage in these specific brain areas has not been reported. Therefore, we must consider that more subtle disturbances involving the neural circuitry of a brain network are involved in the motion processing deficits shown by schizophrenia patients.

Global vs. local processing has been an issue in schizophrenia research, mainly from the Gestalt psychological perspective. In that domain, the processing of global aspects of visual stimuli is concerned with perceptual organization and takes precedence over local processing (Navon, 1977). Studies of schizophrenia patients, however, have been somewhat inconsistent on this score. On the one hand, a general failure by schizophrenia patients in grouping stimuli according to Gestalt aspects of stimuli has been reported (Place and Gilmore, 1980; John and Hemsley, 1992). Another study, however, reported that recognition of objects according to Gestalt principles appeared to be normal in schizophrenia patients (Chey and Holzman, 1997). Still another study indicated that deficient performance could be compensated for by enhancing basic Gestalt properties in the stimuli (Rief, 1991). In the case of motion perception, however, local processing must occur prior to global processing both neuroanatomi- 
cally (e.g. Movshon and Newsome, 1996) and behaviorally (e.g. Adelson and Movshon, 1982). For example, in patients with brain damage involving the striate cortex (responsible primarily for local processing of motion signals), the ability to discriminate the motion direction of complex stimuli, which requires global processing, was compromised (Azzopardi and Cowey, 2001). Thus, the deficits in global motion direction processing in schizophrenia, as shown in this study, cannot be simply accounted for by the global and local processes of classic perceptual organization.

We conclude that motion-sensitive areas of the brain, such as MT, are part of a network that may be implicated in compromised global motion processing in schizophrenia for the following reasons. First, as indicated by normal ability to detect the direction of local motion signals, local motion processing in schizophrenia patients is intact. In terms of underlying neural mechanisms, this result suggests that the primary visual cortex V1 is functionally intact and can be excluded as a major site responsible for the global motion-processing deficit shown in schizophrenia patients. Second, deficits in motion direction processing and in smooth pursuit may occur concurrently or separately, depending on which regions in the individual brain are affected or which parts of the network are involved. For instance, while the cerebellum plays a role in smooth pursuit (e.g., Zee et al., 1981), Nawrot and Razzio (1995) showed that detection of coherent motion is impaired in cerebellum-damaged patients but smooth pursuit is not. This dissociation between motion perception and smooth pursuit in cerebellum, however, does not occur when the motion-sensitive brain area MT is damaged; in MTdamaged monkeys, both smooth pursuit and detection of coherent motion are affected (Newsome and Pare, 1988). Thus, the presence of smooth pursuit impairment and the deficit in detecting coherent motion direction in many schizophrenia patients point to compromised neural mechanisms in the motion-sensitive extrastriate cortex, and perhaps including other projected brain systems with which it interacts. Employing simple moving-target-viewing paradigms, two brain-imaging studies on schizophrenia found inconsistent results. Tost et al. (2001) showed that the dorsal stream of the visual system, where motionsensitive brain areas are located, was significantly less activated in the patients than in the normal controls while Sweeney et al. (2001) showed no significant group activation difference in the same brain areas.

In a previous study (Chen et al., 1998), we used a dynamic random dot pattern and showed that the performances in the tasks of smooth pursuit tracking and of judging the direction of motion were correlated but not identical in normal observers. Indeed, we found little correlation between the directions of the initial eye tracking and the perceived directions of motion when motion signals were relatively weak, yet above the motion detection threshold. Bearing in mind the notion that in normal functioning, smooth pursuit, defined as foveal tracking, and motion perception are closely associated but are different processes, it would now be interesting to see how the smooth pursuit system responds when schizophrenia patients are required to track either local or global motion signals.

\section{Acknowledgements}

We thank the subjects for participation. We thank Ms. Summer Sheremata for testing subjects and Dr. Laurie Terespulsky for recruiting subjects. This research was supported in part by a NARSAD Young Investigator Award, by USPHS Grants MH 61824, $31154,31340,49487,01020$, by a grant from the Roy Hunt Foundation, by a research grant from the William T. Milton Foundation of Harvard University, and by a Rappaport Mental Health Scholar Award.

\section{References}

Adelson, E.H., Movshon, J.A., 1982. Phenomenal coherence of moving visual patterns. Nature 300, 523-525.

Azzopardi, P., Cowey, A., 2001. Motion discrimination in cortically blind patients. Brain 124 (Pt. 1), 30-46, Jan.

Beauchamp, M.S., Cox, R.W., DeYoe, E.A., 1997. Graded effects of spatial and featural attention on human area MT and associated motion processing areas. J. Neurophysiol. 78 (1), 516-520, July.

Bender, D.B., Davidson, R.M., 1986. Global visual processing in the monkey superior colliculus. Brain Res. 381, 372-375.

Berezovskii, V.K., Born, R.T., 2000. Specificity of projections from wide-field and local motion-processing regions within the middle temporal visual area of the owl monkey. J. Neurosci. 20, 1157-1169.

Born, R.T., Tootell, R.B., 1992. Segregation of global and local motion processing in primate middle temporal visual area. Nature 357, 497-499. 
Celebrini, S., Newsome, W.T., 1994. Neuronal and psychophysical sensitivity to motion signals in extrastriate area MST of the macaque monkey. J. Neurosci. 14, 4109-4124.

Chen, Y., McPeek, R.M., Intriligator, J., Holzman, P.S., Nakayama, K., 1998. Smooth pursuit to a movement flow and associated perceptual judgments. In: Becker, W., et al. (Eds.), Current Oculomotor Research: Physiological and Psychological Aspects. Plenum, New York, pp. 125-129.

Chen, Y., Nakayama, K., Levy, D.L., Matthysse, S., Holzman, P.S., 1999a. Psychophysical isolation of a motion-processing deficit in schizophrenics and their relatives and its association with impaired smooth pursuit. Proc. Natl. Acad. Sci. U. S. A. 96, 4724-4729.

Chen, Y., Palafox, G., Nakayama, K., Levy, D., Matthysse, S., Holzman, P., 1999b. Motion perception in schizophrenia. Arch. Gen. Psychiatry $56,149-154$.

Chey, J., Holzman, P.S., 1997. Perceptual organization in schizophrenia: utilization of the Gestalt principles. J. Abnorm. Psychology 106 (4), 530-538, Nov.

Clementz, B.A., Sweeney, J.A., 1990. Is eye movement dysfunction a biological marker for schizophrenia? A methodological review. Psychol. Bull. 108, 77-92.

Collin, N.G., Cowey, A., 1980. The effect of ablation of frontal eyefields and superior colliculi on visualstability and movement discrimination in rhesus monkeys. Exp. Brain Res. 40, 251-260.

Diefendorf, A.R., Dodge, R., 1908. An experimental study of the ocular reactions of the insane from photographic records. Brain 31, 451-489.

Downing, C.J., Movshon, J.A., 1988. Spatial and temporal summation in the detection of motion in stochastic ramdom dot displays. Invest. Ophthalmol. Visual Sci. Suppl. 29, s72.

Dubner, R., Zeki, S.M., 1971. Response properties and receptive fields of cells in an anatomically defined region of the superior temporal sulcus in the monkey. Brain Res. 35 (2), 528-532, Dec. 24.

Friedman, L., Jesberger, J.A., Siever, L.J., Thompson, P., Mohs, R., Meltzer, H.Y., 1995. Smooth pursuit performance in patients with affective disorders or schizophrenia and normal controls: analysis with specific oculomotor measures, RMS error and qualitative ratings. Psychol. Med. 25, 387-403.

Hollingshead, A.B., 1965. Two factor index of social position. Privately printed, 06520, 06520 .

Holzman, P.S., Proctor, L.R., Hughes, D.W., 1973. Eye-tracking patterns in schizophrenia. Science 181, 179-181.

Holzman, P.S., Proctor, L.R., Levy, D.L., Yasillo, N.J., Meltzer, H.Y., Hurt, S.W., 1974. Eye tracking dysfunctions in schizophrenic patients and their relatives. Arch. Gen. Psychiatry 31, $143-151$.

Iacono, W.G., Moreau, M., Beiser, M., Fleming, J.A.E., Lin, T.-Y., 1992. Smooth pursuit eye tracking in first-episode psychotic patients and their relatives. J. Abnorm. Psychology 101, 104116.

John, C.H., Hemsley, D.R., 1992. Gestalt perception in schizophrenia. Eur. Arch. Psychiatry Clin. Neurosci. 241 (4), 215-221.

Kendler, K., 1989. "Structured Interview for Schizotypal Symptoms (SISS, version 1.5)." Department of Psychiatry, Virginia Medical College.
Levy, D.L., Holzman, P.S., Matthysse, S., Mendell, N.R., 1993. Eye tracking dysfunction and schizophrenia: a critical perspective. Schizophr. Bull. 19, 461-536.

Marcar, V.L., Zihl, J., Cowey, A., 1997. Comparing the visual deficits of a motion blind patient with the visual deficits of monkeys with area MT removed. Neuropsychologia 35, 1459-1465.

Merabet, L., Desautels, A., Minville, K., Casanova, C., 1998. Motion integration in a thalamic visual nucleus. Nature 396, $265-$ 268.

Movshon, J.A., Newsome, W.T., 1996. Visual response properties of striate cortical neurons projecting to area MT in macaque monkeys. J. Neurosci. 16, 7733-7741.

Nakayama, K., 1985. Biological image motion processing: a review. Vision Res. 25, 625-660.

Nakayama, K., Silverman, G.H., 1984. Temporal and spatial characteristics of the upper displacement limit for motion in random dots. Vision Res. 24 (4), 293-299.

Navon, D., 1977. Forest before trees: the precedence of global features in visual perception. Cogn. Psychol. 9, 353-383.

Nawrot, M., Razzio, M., 1995. Motion perception deficits from midline cerebellar lesions in human. Vision Res. 35, 723-731.

Newsome, W.T., Pare, E.B., 1988. A selective impairment of motion perception following lesions of the middle temporal visual area (MT). J. Neurosci. 8, 2201-2211.

O’Donnell, B.F., Swearer, J.M., Smith, L.T., Nestor, P.G., Shenton, M.E., McCarley, R.W., 1996. Selective deficits in visual perception and recognition in schizophrenia. Am. J. Psychiatry 153, $687-692$.

Orban, G.A., Saunders, R.C., Vandenbussche, E., 1995. Lesions of the superior temporal cortical motion areas impair speed discrimination in the macaque monkey. Eur. J. Neurosci. 7 (11), 2261-2276, Nov. 1.

Overall, J.E., Gorham, D.R., 1962. Brief psychiatric rating scale. Psychol. Rep., 10.

Place, E.J., Gilmore, G.C., 1980. Perceptual organization in schizophrenia. J. Abnorm. Psychology 89 (3), 409-418, June.

Plant, G.T., Laxer, K.D., Barbaro, N.M., Schiffman, J.S., Nakayama, K., 1993. Impaired visual motion perception in the contralateral hemifield following unilateral posterior cerebral lesions in humans. Brain 116 (Pt. 6), 1303-1335, Dec.

Rief, W., 1991. Visual perceptual organization in schizophrenic patients. Br. J. Clin. Psychol. 30 (Pt. 4), 359-366, Nov.

Rodman, H.R., Albright, T.D., 1989. Single-unit analysis of patternmotion selective properties in the middle temporal visual area (MT). Exp. Brain Res. 75, 53-64.

Rudolph, K., Pasternak, T., 1999. Transient and permanent deficits in motion perception after lesions of cortical areas MT and MST in the macaque monkey. Cereb. Cortex 9 (1), 90-100, Jan.Feb.

Rudolph, K.K., Ferrera, V.P., Pasternak, T., 1994. A reduction in the number of directionally selective neurons extends the spatial limit for global motion perception. Vision Res. 34 (24), 3241 3251, Dec.

Sclar, G., Maunsell, J.H., Lennie, P., 1990. Coding of image contrast in central visual pathways of the macaque monkey. Vision Res. 30 (1), 1-10.

Slaghuis, W.L., 1998. Contrast sensitivity for stationary and drifting 
spatial frequency gratings in positive- and negative-symptom schizophrenia. J. Abnorm. Psychology 107 (1), 49-62, Feb.

Spitzer, R., William, J., Gibbon, M., First, M., 1994. Structured Clinical Interview for DSM-IV-Patient Edition (SCDI-P). American Psychiatric Press, Washington, DC.

Stuve, T.A., Friedman, L., Jesberger, J.A., Gilmore, G.C., Strauss, M.E., Meltzer, H.Y., 1997. The relationship between smooth pursuit performance, motion perception and sustained visual attention in patients with schizophrenia and normal controls. Psychol. Med. 27, 143-152.

Sunaert, S., Van Hecke, P., Marchal, G., Orban, G.A., 2000. Attention to speed of motion, speed discrimination, and task difficulty: an fMRI study. NeuroImage 11 (6 Pt 1), 612-623, June.

Sweeney, J.A., Krisky, C.M., Bureau, A., Keshvan, M.S., 2001. fMRI studies of eye movement control in schizophrenia. Schizophr. Res. 49, NOS 1-2 218, Supplement.

Tanaka, K., Hikosaka, K., Saito, H., Yukie, M., Fukada, Y., Iwai, E., 1986. Analysis of local and wide-field movements in the superior temporal visual areas of the macaque monkey. J. Neurosci. 6 (1), 134-144, Jan.

Tootell, R.B., Reppas, J.B., Kwong, K.K., Malach, R., Born, R.T.,
Brady, T.J., Rosen, B.R., Belliveau, J.W., 1995. Functional analysis of human MT and related visual cortical areas using magnetic resonance imaging. J. Neurosci. 15 (4), 3215-3230, Apr.

Tost, H., Wolf, G., Ruf, M., Henn, F.A., Braus, D.F., 2001. Distributed visual information processing in neuroleptic-native schizophrenic patients: a fMRI study. Schizophr. Res. 49, NOS 1-2 187, Supplement.

Vaina, L.M., Cowey, A., Eskew, R.T., LeMay, M., Kemper, T., 2001. Regional cerebral correlates of global motion perception: evidence from unilateral cerebral brain damage. Brain $124(\mathrm{Pt}$ 2), 310-321, Feb.

Wertheim, A.H., van Gelder, P., Lautin, A., Peselow, E., Cohen, N., 1985. High thresholds for movement perception in schizophrenia may indicate abnormal extraneous noise levels of central vestibular activity. Biol. Psychiatry 20, 1197-1210.

Williams, D.W., Sekuler, R., 1984. Coherent global motion percept from stochastic local motions. Vision Res. 24, 55-62.

Zee, D.S., Yamazaki, A., Butler, P.H., Gucer, G., 1981. Effects of ablation of flocculus and paraflocculus of eye movements in primate. J. Neurophysiol. 46 (4), 878-899, Oct. 